\title{
Autofluorescence imaging of tissue samples using super- high sensitivity fluorescence microscopy
}

\author{
Yuichi Taniguchi* \\ Laboratory for Single Cell Gene Dynamics, RIKEN Quantitative Biology Centre, 6-2-3, Furuedai, Suita, Osaka 565-0874, Japan
}

Single molecule fluorescence microscopy is a technique for superhigh sensitive detection of fluorescence allowing visualization of single molecule fluorescent dyes or proteins on a coverslip [1]. The technique has been artfully used in modern biosciences for a variety of purposes such as super resolution imaging beyond the diffraction limit of light $[2,3]$ and number counting of mRNA or protein molecules $[4,5]$ often in cultured cells on a dish. In this work, I used this microscopy to obtain autofluorescence images of a tissue that is not fluorescently labeled. It is expected that the high sensitivity detection by the microscope allows non-invasive imaging of clear cellular autofluorescence that cannot be detected in conventional fluorescence microscopy, and allows analysis of amounts of specific autofluorescence molecules. In human, it is known that $\mathrm{NAD}(\mathrm{P}) \mathrm{H}$, riboflavin and lipofuscin causes blue, yellow and red autofluorescence, respectively [6-8].

Here, I used a wide-field single molecule fluorescence microscopy, in which a widely expanded laser light is exposed onto the sample, and the emitted fluorescence is detected using a high-sensitivity electron multiplying CCD camera (iXon897, Andor). To obtain a color spectrum of autofluorescence, I performed imaging respectively using four different lasers at 405 (Cube 405, Coherent), 488 (Innova 70C, Coherent), 560 (2RU-VFL-P-2000-560, MPB Communications) and 640 (MRL-III-640-200mW, CNI Laser) nm wavelengths. I used two objective lens with different magnifications: $4 \mathrm{x}$ (UPLFLN 4X, NA = 0.13 , Olympus) and 30x (UPLSAPO 30XS, NA $=1.05$, Olympus).

I imaged tissue from liver (Figure 1), breast (Figure 2), skin of thigh (Figure 3) and foot (Figure 4) of chicken. In liver, I could visualize blood vessels and capillaries at 405, 488 and $560 \mathrm{~nm}$ excitation. The appearance among these wavelengths except for $640 \mathrm{~nm}$ is not much changed, indicating that the autofluorescent molecules have a broad spectrum. In contrast, the image at $640 \mathrm{~nm}$ did not show significant signals compared to the background. This suggests that this wavelength causes less artifacts when we perform imaging of fluorescently labeled molecules targeting on specific proteins or molecules.

Meanwhile, in breast, I could visualize blood vessels as a bright signal and cleavage of muscles as a dark signal (Figure 2). In thigh and foot skin, I could observe patterns of lipid accumulations (Figures 3 and 4). I observed similar fluorescence spectrum tendency to liver, where the images at 405, 488 and $560 \mathrm{~nm}$ excitation are similar, but the image at $640 \mathrm{~nm}$ is not. This imaging itself cannot specify identity of the autofluorescent molecules, and further analyses using mass spectroscopy or immunostaining will provide this kind of information. Accumulation of imaging data with macroscopic symptoms will allow diagnostic analyses of physiological conditions of tissues.
These attempts and results will open the door to the use of ultra-high sensitive fluorescence microscopy for non-invasive and spectroscopic diagnosis in medical tissue samples.

\section{Acknowledgement}

The author thanks Masae Ohno for helpful discussions and David Priest for critically reading the manuscript and editorial assistance. This work was supported by PRESTO, Japan Science and Technology Agency [JPMJPR15F7]; grants-in-aid for Young Scientists (A) [24687022], Challenging Exploratory Research [26650055] and Scientific Research on Innovative Areas [23115005], Japan Society for the Promotion of Science; the Takeda Science Foundation; and the Mochida Memorial Foundation for Medical and Pharmaceutical Research.

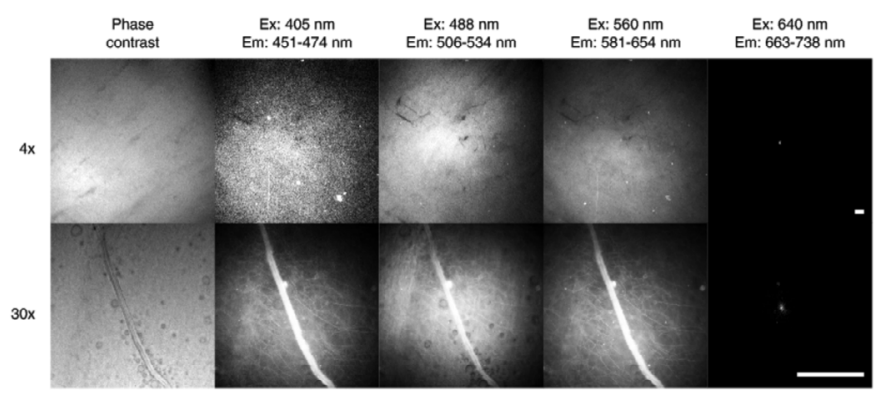

Figure 1. Autofluorescence images of chicken liver. Scale bar: $100 \mu \mathrm{m}$. Ex: excitation wavelength. Em: emission wavelength.

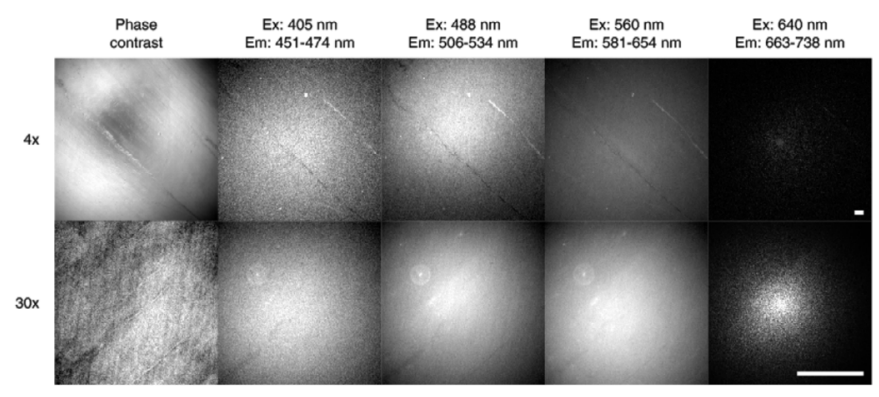

Figure 2. Autofluorescence images of chicken breast. Scale bar: $100 \mu \mathrm{m}$.

Correspondence to: Yuichi Taniguchi, Laboratory for Single Cell Gene Dynamics, RIKEN Quantitative Biology Centre, 6-2-3, Furuedai, Suita, Osaka 565-0874, Japan, E-mail: taniguchi@riken.jp

Received: September 01, 2017; Accepted: September 14, 2017; Published: September 17, 2017 


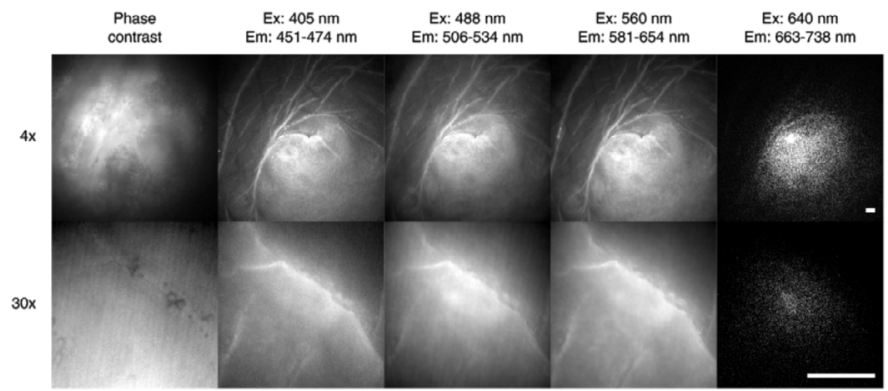

Figure 3. Autofluorescence images of chicken thigh skin. Scale bar: $100 \mu \mathrm{m}$.

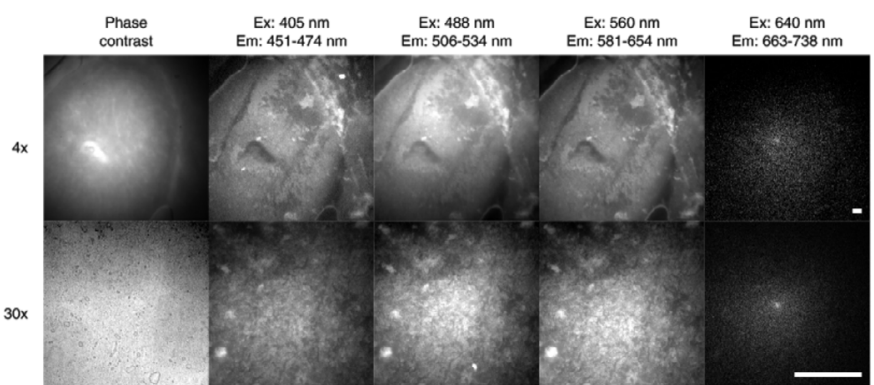

Figure 4. Autofluorescence images of chicken foot skin. Scale bar: $100 \mu \mathrm{m}$.

\section{References}

1. Funatsu T, Harada Y, Tokunaga M, Saito K, Yanagida T (1995) Imaging of single fluorescent molecules and individual ATP turnovers by single myosin molecules in aqueous solution. Nature 374: 555-559. [Crossref]

2. Betzig E, Patterson GH, Sougrat R, Lindwasser OW, Olenych S, et al. (2006) Imaging intracellular fluorescent proteins at nanometer resolution. Science 313: 1642-1645. [Crossref]

3. Rust MJ, Bates M, Zuang X (2006) Sub-diffraction-limit imaging by stochastic optical reconstruction microscopy (STORM). Nat Methods 3: 793-796. [Crossref]

4. Raj A, van den Bogaard P, Rifkin SA, van Oudenaarden A, Tyagi S (2008) Imaging individual mRNA molecules using multiple singly labeled probes. Nat Methods 5: 877879. [Crossref]

5. Taniguchi Y, Choi PJ, Li GW, Chen H, Babu M, et al. (2010) Quantifying E. coli proteome and transcriptome with single-molecule sensitivity in single cells. Science 329: 533-538. [Crossref]

6. Georgakoudi I, Jacobson BC, Müller MG, Sheets EE, Badizadegan K, et al. (2002) $\mathrm{NAD}(\mathrm{P}) \mathrm{H}$ and collagen as in vivo quantitative fluorescent biomarkers of epithelial precancerous changes. Cancer Res 62: 682-687. [Crossref]

7. Zipfel WR, Williams RM, Christie R, Nikitin AY, Hyman BT, Webb WW (2003) Live tissue intrinsic emission microscopy using multiphoton-excited native fluorescence and second harmonic generation. Proc Natl Acad Sci U S A 100: 7075-7080. [Crossref]

8. Schönenbrücher H, Adhikary R, Mukherjee P, Casey TA, Rasmussen MA, et al (2008) Fluorescence-based method, exploiting lipofuscin, for real-time detection of central nervous system tissues on bovine carcasses. J Agric Food Chem 56: 6220-6226. [Crossref]

Copyright: $@ 2017$ Taniguchi Y. This is an open-access article distributed under the terms of the Creative Commons Attribution License, which permits unrestricted use, distribution, and reproduction in any medium, provided the original author and source are credited. 